\title{
Predictors and Prevalence of Central Line Associated Blood Stream Infections Among Adult Patients in Critical Care Units -Kenyatta National Hospital
}

\author{
Mukiri Jocyline ${ }^{1,2,{ }^{*}}$, Inyama Hannah ${ }^{1}$, Maina Dorcas Waithira ${ }^{1}$ \\ ${ }^{1}$ School of Nursing Sciences, University of Nairobi, Nairobi, Kenya \\ ${ }^{2}$ Social Services League M P Shah Hospital, Medical - Surgical Unit, Nairobi, Kenya
}

Email address:

jocylinemukiri@gmail.com (M. Jocyline), hannahinyama@gmail.com (I. Hannah),dwaithira94@gmail.com (M. D. Waithira)

*Corresponding author

\section{To cite this article:}

Mukiri Jocyline, Inyama Hannah, Maina Dorcas Waithira. Predictors and Prevalence of Central Line Associated Blood Stream Infections Among Adult Patients in Critical Care Units -Kenyatta National Hospital. American Journal of Nursing Science.

Vol. 7, No. 1, 2018, pp. 1-13. doi: 10.11648/j.ajns.20180701.11

Received: October 27, 2017; Accepted: December 7, 2017; Published: January 5, 2018

\begin{abstract}
Most adult patients admitted in Critical Care Units (CCUs) have central venous catheters (CVCs). These catheters mostly remain in place for the entire period of hospitalization, hence the risk of developing Central Line Associated Bloodstream Infection (CLABSI). The burden of CLABSI has remained high despite the introduction of CLABSI care bundles increasing the morbidity, mortality, hospital stay and cost. Most CLABSIs are caused by factors attributed to patient characteristics, clinical care and institutional factors. The aim of this study was to determine the prevalence and predictors of CLABSIs among critically ill adult patients at CCUs of Kenyatta National Hospital. The study applied a cross-sectional descriptive design with stratified sampling and simple random sampling for each stratum. 86critical care nurses were selected from a total of 110 nurses using Yamane formulae. Medical records of critically ill patients' that met the inclusion criteria were reviewed for the year 2015. An interviewee administered questionnaire and observation checklist were used to collect data from the nurses, and a data collection sheet was used to collect data from the medical records on prevalence of CLABSIs and patient characteristics. Descriptive statistics was used to summarize the data and inferential statistics (Chi-square test, Pearsons' correlation) was used to establish relationships between variables. Data analysis was done using the Statistical Package for Social Sciences (SPSS) version 21.0. This study revealed that the prevalence of CLABSIs was 3.53\%. Stepwise logistic regression revealed that, the patient predictors of CLABSIs in KNH CCUs were as follows; Neurological disorders as the underlying disease $X^{2}(52)=15.249 ; 95 \%$ CI $-0.199-0.158 ; \mathrm{P}=0.946$, increased length of hospitalization with CVC in situ $\mathrm{X}^{2}(52)=40.639 ; 95 \%$ CI $0.612-0.874 ; \mathrm{P}<0.001$ and parenteral nutrition use $\mathrm{X}^{2}(52)=9.826 ; 95 \%$ CI $0.041-0.759 ; \mathrm{P}=0.013$. In addition, the nursing care related factors that predispose critically ill patients to CLABSIs in KNH CCUs were; Poor practices on hand hygiene before manipulation of infusion line which was observed in $81.8 \%$ of the CCNs, failure to remove unnecessary CVCs promptly, poor knowledge and practices on CVC maintenance and inadequate knowledge and outdated practices on changing intravenous administration system components.
\end{abstract}

Keywords: Central Line Associated Bloodstream Infection (CLABSI), Prevalence, Predictors, Critical Care Units (CCUs), Kenyatta National Hospital (KNH), Critical Care Nurses (CCNs)

\section{Introduction}

\subsection{Background Information}

Central venous catheters (CVCs) use forms an essential part of modern health care worldwide. The National
Healthcare Safety Network (NHSN) defines central venous catheter, or central line, as "an intravascular catheter that ends at or close to the heart or in one of the great blood vessels which is used for hemodynamic monitoring, infusion, or blood sampling." The diagnosis of Central line associated blood stream infection (CLABSI) is key in instituting proper 
management. CLABSI is said to be present if there is a confirmed blood stream infection by laboratory which meets any one of the following criteria in all age groups:

Patient has a recognized pathogenic microbe cultured from one or more blood cultures, which is not related to an infection at another site.

Patient has at least any of these signs or symptoms: fever $\left(>38.0^{\circ} \mathrm{C}\right)$, chills, or hypotension, and the pathogen is not related to infections at any other site or, if the pathogenic microbe is a common commensal, it must be isolated from two or more blood cultures sampled on different occasions [1].

Central line associated blood stream infections (CLABSIs) remain a major complication of central venous catheters and a leading cause of HAIs in the Critical Care Unit (CCU). Estimates are that 80,000 CLABSIs occur in CCU in the United States every year. These CLABSIs not only are costly to health care systems and individuals but can also increase morbidity and mortality significantly in both developed and developing countries [2]. CLABSIs are serious infections but often preventable when evidence-based guidelines such as CLABSI care bundle are used during the insertion and maintenance of central venous catheters (CVCs) [3]. The care bundles incorporate the most basic infection prevention and control practices which can be practiced even in resource limited settings. Application of the preventive measures such as, use of this CLABSI care bundle, during insertion and maintenance of the central lines, has been shown to remarkably reduce the incidence and prevalence of CLABSIs. The health care providers are charged with the insertion and maintenance of the central venous catheters. There have been inconsistencies in the handling, aseptic techniques application during insertion and management of these venous accesses leading to continued occurrences of CLABSI. Although adherence to evidence-based practices can significantly improve patient safety and quality of care and lowers inconsistencies in practice, health care organizations often find it hard to implement best practices, meeting various barriers that hinder their success [4]. The reason for this varies depending on the setting. Identification and removal of barriers to adherence to these practices is necessary for a successful implementation strategy of the CLABSI care bundle [5]. In resource-limited countries, CLABSIs rates are up to 44.6 cases per 1,000 central venous catheter days in adult intensive care units and up to 60 cases per 1,000 central venous catheter days in neonatal ICUs. These infections were linked to significant extra mortality [6]. The purpose of this study was therefore to describe the predictors and prevalence of CLABSI at the KNH CCUs.

\subsection{Statement of the Problem}

Central venous catheters (CVCs) are inserted in most of $\mathrm{CCU}$ patients and this makes them vulnerable to CLABSIs. In the United States, 15 million CVC days occur in CCUs each year [1]. It is estimated that in Europe, about 4.1 million patients develop HAIs (CLABSIs among them) [7]. CLABSIs are associated with an increased risk of morbidity and mortality, medical costs and length of hospitalization.
They result in 16 million added hospital days and over 37,000 attributable mortalities, and they contribute to more than 110,000 extra deaths in Europe each year. In the United States, the prevalence of HAIs including CLABSIs was $4.5 \%$ whereas that of Korea was 3.7\% [8]. In these developed countries, HAIs are expected to be non- existent or very few, although they continue to be a huge burden. In another study, it was observed that the prevalence of HAIs including CLABSIs in Tunisia and Morocco was $17.8 \%$ whereas that of Mali was $18.7 \%$ and in Tanzania it was $14.8 \%$. This shows higher HAIs prevalence rates in developing than in developed countries [8]. Similarly, a study done in Kenya at $\mathrm{KNH}$ CCU estimated the CLABSI prevalence rate at $12 \%$ [9]. This rate compares with other rates in developing countries. The studies show that CLABSIs are a major problem both in the developed and developing countries. There is a significant gap on the CLABSIs burden globally, regionally and locally with regional and local areas experiencing higher burden. In $\mathrm{KNH} \mathrm{CCU}$, the number of overall patients with central lines is high hence the vulnerability of these patient populations to CLABSIs. This disparity between developed and developing countries is attributed to: limited training and knowledge in basic infection prevention and control, poor adherence to routine hand hygiene, limited resources, reuse of equipment (e.g. needles, gloves), inappropriate and prolonged use of antimicrobials and invasive devices, understaffing, limited local and national policies, and limited guidelines [10]. Treatment of these infections requires resources which are limited in Kenya. Critically ill patients with CVCs are expected to be CLABSI free. On the contrary, anecdotal evidence suggests that these infections are rampant and contribute to increased medical costs, prolonged hospitalization, and increased morbidity and mortality. Despite this heavy burden, there is no study that has been done locally to evaluate the factors contributing to CLABSI occurrence. Patient characteristics, clinical care factors and institutional factors have been found to increase the risk. This study therefore sought to describe the factors that contribute to CLABSI at CCUs KNH.

\subsection{Justification}

Kenyatta National Hospital (KNH) is the main referral hospital in Kenya and has the highest number of critically ill patients in the country. Most of these patients are vulnerable to CLABSIs due to the fact that they are critically ill and have a central venous catheter in place. Central line associated blood stream infections (CLABSIs) are associated with an increased risk of mortality and morbidity, cost and prolonged hospital stay. Due to the advancement in technology leading to easier access of information nurses are expected to be more knowledgeable regarding patient care and infection prevention measures. Also, the introduction of CLABSI clinical practice guidelines (CPGs) by the Institute of Health Care Improvement in 2009 was expected to lead to tremendous decline in CLABSI prevalence rates. The investigator therefore found the need to determine the impact 
of advanced technology and CLABSI CPGs by ascertaining the difference in prevalence rates of CLABSI as at 2015 and the previous CLABSI prevalence rate of $12 \%$ at 2007 . For infections occurrence, patients' inherent characteristics and the environment play a major role. The investigator thus determined the predictors of CLABSIs among the critically ill patients at $\mathrm{KNH}$ CCUs in terms of patient characteristics and nursing care related factors that influence the occurrence of these infections which are vital in their prevention. Currently there is no evidence of studies conducted in $\mathrm{KNH}$ CCUs to determine the predictors of CLABSIs among critically ill adult patients. This necessitated the need to conduct a scientific study in this area of patient care as the information obtained from this study would shed light on factors to be given more consideration in prevention of CLABSI. It would also form a valuable guide to nursing practice, policy formulation and curriculum development on prevention of CVC infections among critically ill patients. It would also provoke more research in CVC related infections in the critically ill patients.

\subsection{Objectives}

The broad objective was to determine the prevalence and predictors of CLABSIs among critically ill adult patients in CCUs KNH.

The specific objectives were:

a. To determine the prevalence of CLABSIs among critically ill adult patients in CCUs KNH.

b. To determine the characteristics of critically ill adult patients with CLABSIs, in terms of their risk factors, in CCUs KNH.

c. To identify nursing care related factors that predisposes critically ill adult patients to CLABSIs in CCUs KNH.

\section{Literature Review}

\subsection{Prevalence of Central Line Associated Blood Stream Infections (CLABSI)}

Available data on the global influence of health care associated infections (HAIs) have been scanty, especially in many resource-limited areas. Low and middle income countries lack enough resources generally to conduct HAIs surveillance. This represents a significant gap, as 144 out of 209 countries are ranked by the World Bank as developing countries accounting to more than $75 \%$ of the world population [11]. However, researchers who have aimed at quantifying HAI rates (CLABSIs among them) in developing countries have found rates to be much on the higher side than in developed countries [11], and their effects on patients and health care delivery systems is severe and underestimated. These same HAIs are much lower in developed countries. In Europe the average prevalence is 7.1 per 100 patients while the rate is at 4.5 per 100 patients in the United States of America [12]. In comparison the rates in the developing countries are much higher. It is estimated that the rate is 47.9 with an incidence estimation of 13.6 per 1000 patient days
[12]. This disparity could be attributed to differences in resources. The commonest cause of CLABSI is gram positive bacteria where Coagulase-negative staphylococci and Staphylococcus aureus respectively account for $31 \%$ and $20 \%$ of HAIs and Enterococcus and Candida species were in position third and fourth, at $9 \%$ each. Gram-negative organisms caused one quarter of the infections with Escherichia coli $(6 \%)$ and Klebsiella species being the most frequent [12]. Organisms of Gram negative origin, though, have been established to be a more significant cause of CLABSIs in some other areas of the world. Taiwan reported $50 \%$ and Egypt reported $66 \%$ of CLABSIs more often due to Gram negative organisms most frequently due to Escherichia coli, Klebsiella pneumoniae, and Pseudomonas aeruginosa [12]. This is comparable to the study done in Kenya where the prevalence of CLABSI was at $12 \%$ and gram negative organisms (10 out of 12 organisms), with Klebsiella species as the leading organisms, was responsible for CLABSIs [9].

\subsection{Patient Characteristics That Influence Occurrence of CLABSI}

Patient physiological characteristics can predispose them to CLABSI. Extreme ages pose greater risk of CLABSI associated with weakened immune system. The gender of the patient may also play a role in CLABSI. In Geneva, male gender was identified as a characteristic linked to increased risk of CLABSI $[4,13]$. The underlying diseases or condition can also increase the risk. Hematological, immunological, cardiovascular and gastrointestinal diseases are associated with increased risk of infection [14]. These conditions decrease the patient's ability to fight infections. These findings compare to other studies which showed a 2.7 higher CLABSI risk in patients with gastrointestinal diagnoses as compared with patients who had respiratory diagnoses [15]. The more severe the illness the higher the likelihood of the patient having multiple CVCs and also prolonged use of these devices. There was a tenfold rise in risk of CLABSI in CCU patients with more than one CVC [16]. An increase in CVC dwell time increases CLABSI risk. The length of hospitalization with CVC in situ impacts CLABSI occurrence. In another study, the daily CLABSI risk was very low in patients during the first 7 days of catheterization but doubled thereafter [15]. It is expected that the longer the $\mathrm{CVC}$ is in situ the more the exposure to microorganisms and the chances of infection. Further these critically ill patients may be unable to feed enterally and the CVC may be used for parenteral feeding. This increases the risk of infection since these solutions can favor microbial growth. Lipid emulsion is the most likely component of parenteral nutrition to foster fungal or bacterial proliferation [14]. The risk is therefore higher among parenterally fed patients.

\subsection{Nursing Care Related Factors: Nurses' Knowledge and Practices on CVC Use That Impact on CLABSI Occurrence}

Hand hygiene is a major part of any effective infection 
prevention and control and patient safety program. Both alcohol-based hand rub products and soap and water can be used to achieve proper hand hygiene [17]. It is accepted generally as the single most important precaution in spread of infection prevention. It is crucial that health care personnel have knowledge on the practices recommended for hand hygiene and that they adhere to them consistently [8]. Incorporation of hand hygiene in CVC care bundles is important. It is recommended that organizations incorporate hand hygiene into procedures done routinely and put in place strong systems to monitor, support and promote the correct behavior [10]. The impact of hand hygiene on HAIs has been extensively studied. In Switzerland, hand hygiene was found to decrease the HAIs from a baseline of $16.9 \%$ to $9.9 \%$ [12]. This was following a hand hygiene promotion program. In a similar study, targeting proper hand hygiene and CVC care, it was found that the rates of CLABSI decreased from 3.9 to $1.0 / 1000$ CVC days after the intervention [4]. In order to decrease the risk of CLABSI associated with direct contact of the hands of health care providers, guidelines recommend that hand hygiene be performed at the following times: before and after palpating the site of $\mathrm{CVC}$ insertion; before and after inserting the $\mathrm{CVC}$; and before and after accessing, dressing, repairing, or replacing the CVC [1].

Microbial colonization reduction at the site of CVC insertion is a crucial component of preventing CLABSIs. Chlorhexidine has been found to be superior in the prevention of CLABSI [18]. Cleansing the site with Chlorhexidine reduces the colonization of the skin by microbes that would otherwise infect the catheter. An analysis of 4000 catheters found that chlorhexidine reduced the risk of CLABSI by almost $50 \%$ in comparison to the use of povidone-iodine [19]. It was demonstrated that chlorhexidine effects a greater reduction in skin flora for a longer duration of time than povidone-iodine and is not inactivated by serum or blood proteins [20]. In some countries where chlorhexidine availability may be a problem however, povidone-iodine should be used [6]. It is preferred to not using any skin antiseptic agent.

The siting of the catheter determines whether it is prone to contamination. Data obtained from several observational studies of CVC insertions, indicate that the highest risk of CLABSI in adults is linked to femoral vein use as the site of insertion, and the lowest risk is linked to subclavian insertion sites, with an intermediate level of risk associated with internal jugular vein insertions for non-tunneled CVCs. This risk is attributed to the colonization density of skin flora at the site of CVC insertion [21].

The CLABSI risk increases with the duration of time the CVC is left in situ. Therefore, daily review of the continued need for a CVC is a significant aspect of CLABSI prevention. CVCs that are no longer necessary should be removed promptly [22]. Leaving them in situ increases the risk of exposure to nosocomial infections. $4.8 \% \mathrm{CVC}$ days were found to be unnecessary [23]. The patient did not need the CVC for any indications that could be identified by the nurse or the doctor. In another study, it was found out that
$4.6 \%$ catheter days were not justified [24]. Daily reviews of the continued necessity for CVCs can be carried out during patient care rounds by multidisciplinary teams or by using reminders, e.g. stickers on patient records, or via computer alerts [10]. This helps the staff identify the need for the CVC and thus its prompt removal when not needed.

A dry and clean dressing at the CVC insertion site is key to protecting the site and to reducing the risk of CLABSI [17]. The evidence-based recommendations concerning CVC site dressing regimens are: use a sterile, transparent semipermeable dressing or sterile gauze to cover the CVC insertion site; use a gauze dressing if the site is oozing or the patient is diaphoretic; replace the dressing if it becomes visibly soiled, damp or loosened; replace gauze dressings every two days; and replace semipermeable dressings every seven days, and more frequently if loose, soiled or damp [21]. It is also vital that the CVC insertion site be inspected via a dressing that is intact. Presence of fever with no obvious source, tenderness at the CVC insertion site, or other symptoms suggestive of either local or bloodstream infection prompts the dressing to be removed and the site thoroughly inspected [17].

The cumulative risk of contamination of an intravenous (IV) system increases if an infusion runs for an extended period. Hence, it is important that the IV infusion system, which comprises the primary and any secondary sets and add-on devices, be replaced on a regular basis. Current evidence suggests that the most appropriate interval for routine replacement of continuous intravenous administration sets is no more frequently than every 96 hours, and at least every 7 days, after beginning of its use. This interval of replacement is safe and permits substantial cost savings to health care institutions. However, if fluids that foster microbial growth are infused (e.g. parenteral feeds preparations and Human Albumin) administration sets and add-on devices should be replaced within 24 hours of the initiation of infusion and intermittent sets should be replaced every 24 hours [25]. Needleless components should be changed at least as often as the administration set and no more often than every 96 hours [17].

\subsection{Theoretical Framework}

The framework that was adopted for this study was 'Levels of Prevention Model' by Leavell and Clark, 1975 which has been defined in terms of four levels; Primordial prevention-This entails prevention of the emergence or development of risk factors in population in which they have not yet appeared. Primary prevention- This includes actions taken prior to the onset of disease, which removes the possibility that the disease will ever occur. Its strategies emphasize general health education and health promotion, risk factor reduction, and environmental health programs designed to improve environmental quality. Secondary prevention- This includes actions that stop the progress of a disease at its initial stage. It focuses on individuals who are experiencing illness and are at risk of developing complication. Activities are directed at early diagnosis and 
prompt intervention, hence reducing severity and enabling the individual to return to normal. Its purpose is to cure disease, slow disease progression, or reduce its impact on individuals or communities. These strategies are expensive and less effective than primary prevention. Tertiary prevention- This occurs when a disability is permanent and irreversible. Preventive strategies are aimed at minimizing the effects of long-term disease or disability. The interventions are directed at preventing complications and deterioration. Here, strategies are both therapeutic and rehabilitative measures once disease is firmly established. This study sought to determine predictors of CLABSIs which when taken into consideration during the primordial prevention level will curb occurrence of these infections. Determining the health care providers' knowledge and practices on CVC use and care would shed light on removing the possibility that CLABSIs will ever occur and this is during primary prevention. In secondary CLABSI prevention, thorough inspection of CVC insertion site when signs and symptoms suggesting local or bloodstream infection will point to early diagnosis and prompt intervention hence reducing morbidity rates and costs. Tertiary CLABSI prevention aims at reducing complications and mortality rates from this infection. The implementation of the recommendations from the results of this study will greatly reduce the likelihood that CLABSIs will progress to this tertiary level of prevention.

\section{Research Methods}

This was a cross-sectional research design utilizing mixed methods. It incorporated a retrospective review of patient records and observation of the CVC care bundle implementation among the nurses. The retrospective design was used since the data collection period was two months. A quantitative approach was followed to describe interactions among study variables. The study population comprised of critically ill adult patients medical records admitted at $\mathrm{KNH}$ CCUs over the year 2015 and had a central venous catheter at any point of time during their admission in CCU. Nurses working at the $\mathrm{KNH}$ CCUs on permanent employment and consented to participate in the study also comprised the population in this study. A sample of 86 nurses determined using Yamane formula from a total population of 110 was used to obtain data on knowledge of nurses while a sample of 11 nurses obtained from $10 \%$ of total population comprised the sample under observation on practices of CVC use and maintenance. A sample of 52 medical records was used to obtain data on patient characteristics and was obtained using Yamane Formula from a monthly total of 60 admissions to KNH CCUs. Stratified sampling was used to select the required sample and the strata consisted of the senior nursing officers, nursing officer ones, nursing officer twos and nursing officer threes. Simple random sampling was then used to select the required sample from each stratum.

A questionnaire, an observation checklist and data sheets was used to collect data for this study. A data sheet was used to obtain data on prevalence of CLABSI and patient characteristics from the medical records of the critically ill adult patients. A questionnaire was used to collect data from nurses working in $\mathrm{KNH}$ CCUs on their knowledge on CVC insertion and maintenance bundles. It had sections on knowledge and practice. The questionnaire was prepared by the researcher based on the $\mathrm{CVC}$ bundle. It was reviewed by experts in critical care $(n=3)$ for validity. A participant observation checklist was used to obtain data from the nurses working at $\mathrm{KNH}$ CCUs on their practice on CVC use and care. The study was approved by Kenyatta National Hospital/University of Nairobi Ethics and Research Committee (KNH/UON ERC). Clearance to conduct research was sought from KNH authorities. Data on patient characteristics such as age, gender, medical condition, days with CVC in situ, parenteral nutrition and antibiotic use was collected from 52 selected patients' medical records with the researcher filling in this information in a data collection sheet. Data on prevalence of CLABSI was obtained by reviewing the medical records of patients who had been admitted to CCUs over the year 2015 and had a CVC inserted and this information filled in the data collection sheet. Informed consent was obtained from 86 selected nurses working in KNH CCUs and a questionnaire was then issued for them to fill in their knowledge on CVC use and care. The researcher ensured that the questionnaire was completely filled and returned. Participant observation of the practice level among 11 nurses working at $\mathrm{KNH}$ CCUs on CVC use and care was done by the researcher and an observation checklist with specific variables derived from the elements of the CVC maintenance bundle was filled immediately after. Each nurse was observed three times to account for Hawthorne effect. An average of the observations was then calculated to determine the actual practice on CVC use and care. Out of the three observations those who performed the elements twice and above were considered to be having good practices while those who just performed the elements once out of the three observations were considered to be having poor practices. A score $\geq 70 \%$ was considered knowledgeable and good practice. A score $<70 \%$ was considered non- knowledgeable and poor practice. These scores were agreed upon by experts in critical care $(n=3)$. At the point of collection, data was organized, examined for accuracy and completeness, coded and entered into computer software SPSS version 21.0 ready for analysis. The raw data in form of questionnaires, checklists and data sheets was kept in a safe under lock and key while processed data is stored in a flash disk, in a password protected folder.

\section{Results}

\subsection{Prevalence of CLABSIs Among Critically Ill Adult Patients Admitted in KNH CCUs in 2015}

A total of 474 patients' medical records were reviewed. 283 of these recorded presence of CVCs and 10 patients developed CLABSIs. The prevalence of CLABSIs among 
critically ill adult patients admitted in KNH CCUs in 2015 was therefore $3.53 \%$. The microorganisms identified for causing CLABSIs according to blood culture reports included: klebsiella pneumonia, Enterococcus, pseudomonas aeruginosa, escherichia coli and cougulase negative staphylococci. Klebsiella pneumonia was the commonest at $60 \%$, with the others having an equal chance at $10 \%$ each.

\subsection{Characteristics of Critically IIl Adult Patients with CLABSIs, in Terms of Their Risk Factors, in KNH CCUs in 2015.}

\subsubsection{Patient's Characteristics}

Table 1. Patient's characteristics.

\begin{tabular}{|c|c|c|c|c|}
\hline \multirow{3}{*}{ Characteristic } & \multirow{2}{*}{ Frequency } & \multirow{2}{*}{ Percentage } & \multicolumn{2}{|l|}{ CLABSI } \\
\hline & & & Present & Absent \\
\hline & $\mathbf{n}=\mathbf{5 2}$ & $(\%)$ & n (\%) & n (\%) \\
\hline \multicolumn{5}{|l|}{ Age in years } \\
\hline $18-27$ & 13 & 25 & $4(30.8 \%)$ & $9(69.2 \%)$ \\
\hline $28-37$ & 17 & 32.7 & $2(11.8 \%)$ & $15(88.2 \%)$ \\
\hline $38-47$ & 9 & 17.3 & $2(22.2 \%)$ & $7(77.8 \%)$ \\
\hline $48-57$ & 7 & 13.5 & $0(0.0 \%)$ & $7(100.0 \%)$ \\
\hline $68-77$ & 4 & 7.7 & $1(25.0 \%)$ & $3(75.0 \%)$ \\
\hline \multicolumn{5}{|c|}{ Mean Age (+SD) 38.7(14.7), range $18-74$ years } \\
\hline \multicolumn{5}{|c|}{ Gender } \\
\hline Male & 38 & 73.1 & $7(18.4 \%)$ & $31(81.6 \%)$ \\
\hline Female & 14 & 26.9 & $3(21.4 \%)$ & $11(78.6 \%)$ \\
\hline \multicolumn{5}{|c|}{ Underlying condition/ disease } \\
\hline Hematologic disease & 3 & 5.8 & $0(0.0 \%)$ & $3(100.0 \%)$ \\
\hline Gastrointestinal disease & 6 & 11.5 & $2(33.3 \%)$ & $4(66.7 \%)$ \\
\hline Neurological disorders & 14 & 26.9 & $7(50.0 \%)$ & $7(50.0 \%)$ \\
\hline Genital urinary disease & 4 & 7.7 & $0(0.0 \%)$ & $4(100.0 \%)$ \\
\hline Musculoskeletal disease & 7 & 13.5 & $0(0.0 \%)$ & $7(100.0 \%)$ \\
\hline Respiratory disease & 8 & 15.4 & $1(12.5 \%)$ & $7(87.5 \%)$ \\
\hline \multicolumn{5}{|l|}{ CVC insertion site } \\
\hline Subclavian vein & 38 & 73.1 & $9(23.7 \%)$ & $29(76.3 \%)$ \\
\hline Femoral vein & 1 & 1.9 & $0(0.0 \%)$ & $1(100.0 \%)$ \\
\hline Internal jugular vein & 13 & 25 & $1(7.7 \%)$ & $12(92.3 \%)$ \\
\hline \multicolumn{5}{|l|}{ Parenteral nutrition use } \\
\hline Yes & 6 & 11.5 & $4(66.7 \%)$ & $2(33.3 \%)$ \\
\hline No & 46 & 88.5 & $6(13.0 \%)$ & $40(87.0 \%)$ \\
\hline \multicolumn{5}{|c|}{ Systemic antimicrobial therapy } \\
\hline None & 2 & 3.8 & $1(50.0 \%)$ & $1(50.0 \%)$ \\
\hline Penicillin & 9 & 17.3 & $1(11.1 \%)$ & $8(88.9 \%)$ \\
\hline \multicolumn{5}{|l|}{ Number of days with CVC } \\
\hline $1-7$ & 27 & 52 & $0(0.0 \%)$ & $27(100.0 \%)$ \\
\hline $8-14$ & 17 & 36.5 & $2(11.8 \%)$ & $15(88.2 \%)$ \\
\hline 15 and above & 8 & 11.5 & $8(100.0 \%)$ & $0(0.0 \%)$ \\
\hline Mean Days(+SD) 9.2(5.7 & & & & \\
\hline
\end{tabular}

A total of 52 patients were sampled. The patients characteristics put into consideration in this study were age, gender, underlying disease, CVC insertion site, parenteral nutrition use, systemic antimicrobial therapy and number of days with CVC. Male patients (73.1\%) and those aged between 28 and 37 years $(32.7 \%)$ were the majority. Patients with neurological disorders $(26.9 \%)$ were the most studied. On the CVC insertion site, Subclavian vein $(73.1 \%)$ was the insertion site of choice whereas femoral vein $(1.9 \%)$ was least preferred. Patients on parenteral nutrition were fewer $(11.5 \%)$. Patients not on systemic antibiotics were $(3.8 \%)$ and cephalosporins were the antibiotics mostly used (69.2\%). $51.9 \%$ of patients had a CVC in situ for seven days or less.
These results are summarized in Table 1

The patient age group where CLABSI occurred the most was 18-27 years (40.0\%). The least affected age group was 48-57 years whereby no CLABSI was identified. Among the ten patients who developed CLABSI, seven $(70 \%)$ were male. Female patients were least affected where only $3(30 \%)$ developed CLABSI. Patients with neurological disorders were the most affected by CLABSI (70\%). Gastrointestinal disease followed at $20 \%$ and then respiratory system disease at $10 \%$. Patients with other underlying conditions did not experience CLABSIs. Patients with CVCs at subclavian veins had more CLABSIs (90\%) while internal jugular CVCs had $10 \%$ CLABSIs. Femoral vein CVCs did not have 
CLABSIs. Six patients were on parenteral nutrition and out of them, $66.7 \%$ developed CLABSIs. 46 patients were not on parenteral nutrition but $13 \%$ of them developed CLABSIs.
Patients on cephalosporins experienced more CLABSIs $(60 \%)$. Patients not on any antibiotic had an equal chance of developing CLABSI. These results are shown in Figure 1.

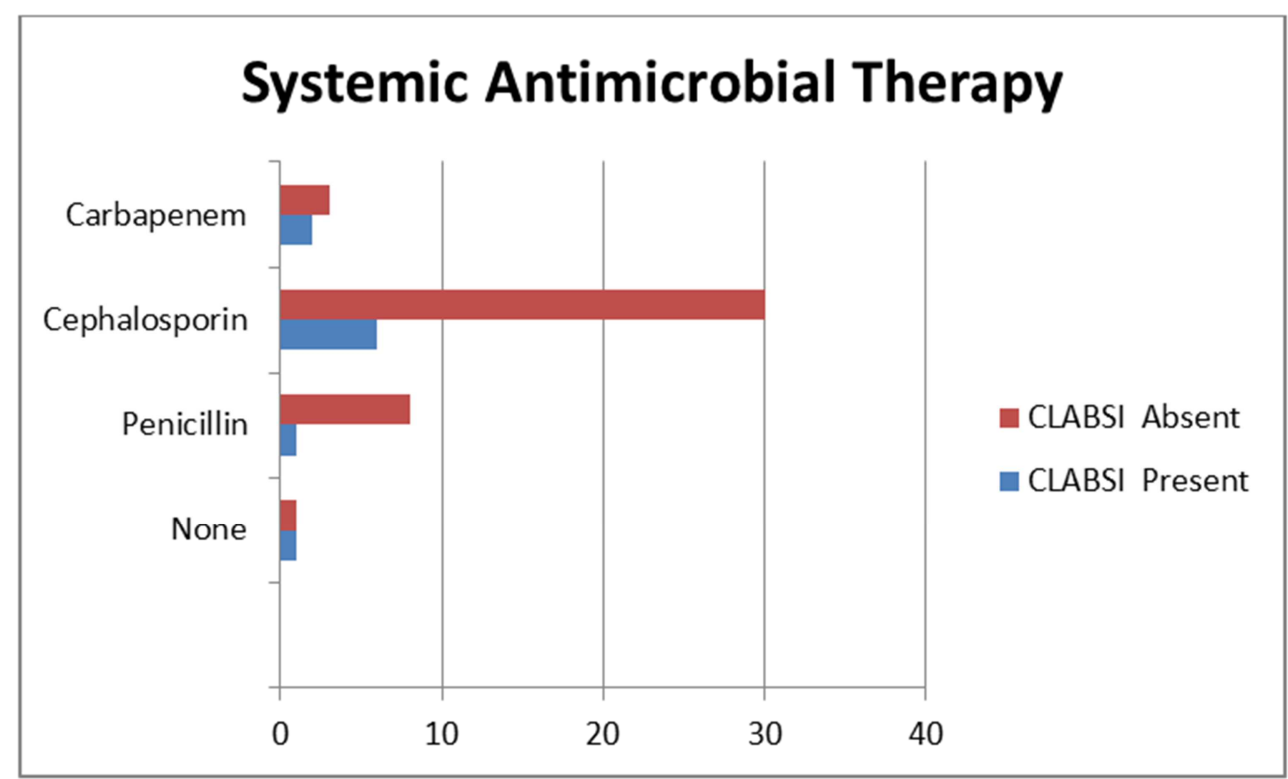

Figure 1. Systemic antimicrobial use versus CLABSI occurrence.

Most Patients with CLABSI (80\%) had a CVC in situ for more than 14 days. Those who had a CVC for 7 days or less did not develop CLABSI as displayed in Figure 2.

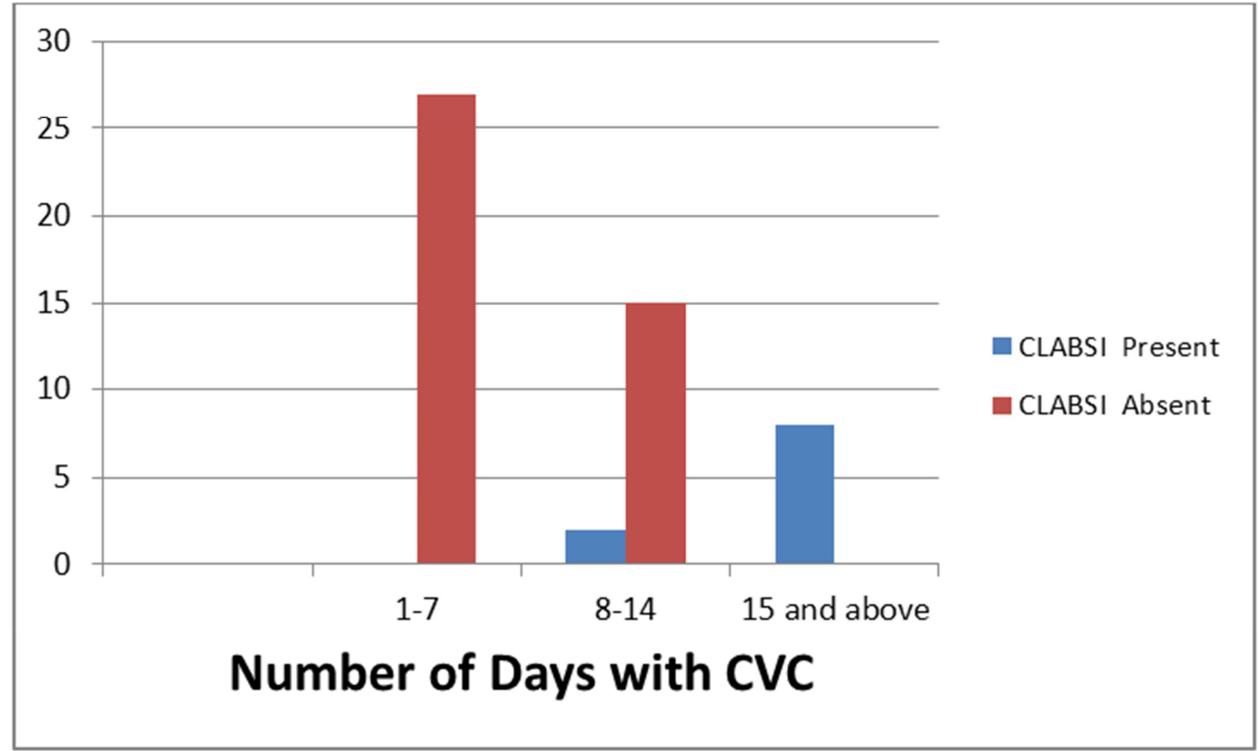

Figure 2. Number of days with CVC versus CLABSI occurrence.

\subsubsection{Association of Patient's Characteristics and CLABSI}

To generate P- values, characteristics were tested against; age- 28-37 years, gender- male, underlying disease- central nervous system, CVC site- subclavian, number of CVC days 1-7, parenteral nutrition use- no, systemic antibiotic usecephalosporin. The results are summarized in Table 2 .

These results show that there is an association between gender and CLABSI occurrence $\mathrm{P}<0.001$. The male gender was the most affected by CLABSI. There was a relationship between underlying condition and CLABSI occurrence $\mathrm{x}^{2}=15.249$. Patients with neurological disorders were most affected. Parenteral nutrition use was associated with increased CLABSI occurrence $\mathrm{P}=0.013$. There was a strong positive correlation between CLABSI and number of days with $\mathrm{CVC}^{2}=0.774$. Thus an increase in number of days with $\mathrm{CVC}$ led to an increase in CLABSI risk. 
Mukiri Jocyline et al.: Predictors and Prevalence of Central Line Associated Blood Stream Infections Among

Adult Patients in Critical Care Units -Kenyatta National Hospital

Table 2. Association of patient's characteristics and CLABSI.

\begin{tabular}{|c|c|c|c|c|c|c|c|c|}
\hline \multirow{2}{*}{ Predictor } & \multirow{2}{*}{$\mathbf{R R}$} & \multicolumn{2}{|c|}{$95 \% \mathrm{CI}$} & \multirow{2}{*}{$\mathrm{X}^{2}$ Value } & \multirow{2}{*}{$d f$} & \multirow{2}{*}{$\frac{X^{2} \text { Test }}{\text { P-value }}$} & \multirow{2}{*}{$\begin{array}{l}\text { Linear- by- linear } \\
\text { association }\end{array}$} & \multirow{2}{*}{$\mathbf{r}^{2}$} \\
\hline & & Lower & Higher & & & & & \\
\hline Age & 5.744 & -0.297 & 0.313 & 4.748 & 5 & 0.067 & 0.75 & 0.038 \\
\hline Gender & 0.59 & -0.305 & 0.228 & 0.06 & 1 & 0.000 & 0.58 & -0.034 \\
\hline Underlying condition/ disease & 17.839 & -0.199 & 0.158 & 15.249 & 7 & 0.946 & 0.019 & -0.019 \\
\hline CVC insertion site & 2.259 & -0.045 & 0.357 & 1.838 & 2 & 0.000 & 1.651 & 0.18 \\
\hline Parenteral nutrition use & 7.652 & 0.041 & 0.759 & 9.826 & 1 & 0.013 & 9.637 & 0.435 \\
\hline Systemic antimicrobial therapy & 2.691 & -0.363 & 0.272 & 3.142 & 3 & 0.088 & 0.088 & -0.042 \\
\hline No. of days with CVC & 38.598 & 0.612 & 0.874 & 40.639 & 2 & 0.000 & 30.564 & 0.774 \\
\hline
\end{tabular}

Key: RR- Relative Risk, CI- Confidence Interval, $\mathrm{X}^{2}$ - Chi Square, $\mathrm{df}$ - degree of freedom, $\mathrm{r}^{2}$ - Pearson Correlation.

\subsection{Nursing Care Related Factors That Put Critically Ill Patients in KNH CCUs at Risk of Developing CLABSI}

\subsubsection{Socio- Demographic Data of CCNs}

The socio- demographic data of CCNs studied was age, gender, highest education certificate, cadre and experience in CCU. Most of the nurses enrolled in the study were aged between 31-40 years (55.8\%). Female CCNs $(73.3 \%)$ formed the majority of those enrolled in the study. KRCCNs were enrolled most in the study $(73.3 \%)$. SNOs were the highest enrolled in the study $(57.0 \%)$. CCNs with $<3$ years' experience were least enrolled in the study $(10.5 \%)$ as summarized in Table 3.

Table 3. Socio- demographic data of CCNs.

\begin{tabular}{lll}
\hline Demographic data & Frequency (n=86) & Percentage\% \\
\hline Age in years & 16 & 18.6 \\
$21-30$ & 48 & 55.8 \\
$31-40$ & 18 & 20.9 \\
$41-50$ & 4 & 4.7 \\
$51-60$ & & \\
Mean (+SD) 37.5(7.53)Range 23-56 years & & 26.7 \\
Gender & 23 & 73.3 \\
Male & 63 & 4.7 \\
Female & & 73.3 \\
Highest education certificate & 4 & 22.1 \\
KRCHN & 63 & \\
KRCCN & 19 & 57 \\
BSN & & 4.7 \\
Cadre & 49 & 33.7 \\
SNO & 4 & 4.7 \\
NOIII & 29 & 10.5 \\
NOII & 4 & 45.3 \\
NOI & & 24.4 \\
Experience in CCU & 9 & 19.8 \\
$<$ 3years & 39 & \\
3-6years & 21 & \\
7-10years & 17 & \\
10+ years & & \\
Mean (+SD) 6.7(3.68) & & \\
\hline
\end{tabular}

\subsubsection{Knowledge Level of CCNs on CVC Handling}

Knowledge was measured by asking questions on hand hygiene, skin antisepsis, prompt removal of unnecessary CVCs, proper CVC maintenance and changing IV administration system components. Poor knowledge was interpreted as a score of below 70\%. The scores cut off was agreed upon by experts in critical care $(n=3)$. The results are summarized in table 4 .

Table 4. Knowledge level of CCNs on CVC handling.

\begin{tabular}{lll}
\hline Variable/knowledge level & \% good knowledge & \% poor knowledge \\
\hline Hand hygiene & 67.4 & 32.6 \\
Skin antisepsis & 90.7 & 9.3 \\
Prompt removal of unnecessary CVCs & 83.7 & 16.3 \\
Proper CVC maintenance & 14 & 86 \\
Changing IV administration system components & 50 & 50 \\
\hline
\end{tabular}


These results show that most CCNs had good knowledge on hand hygiene, skin antisepsis, and prompt removal of unnecessary CVCs where $67.4 \%, 90.7 \%$ and $83.7 \%$ of them scored $70 \%$ and above on these variables respectively. CCNs had poor knowledge on proper CVC maintenance where only $14 \%$ of them scored $70 \%$ and above on this variable.

CCNs who were generally knowledgeable on all aspects of CVC handling were $36 \%$ as shown in Figure 3.

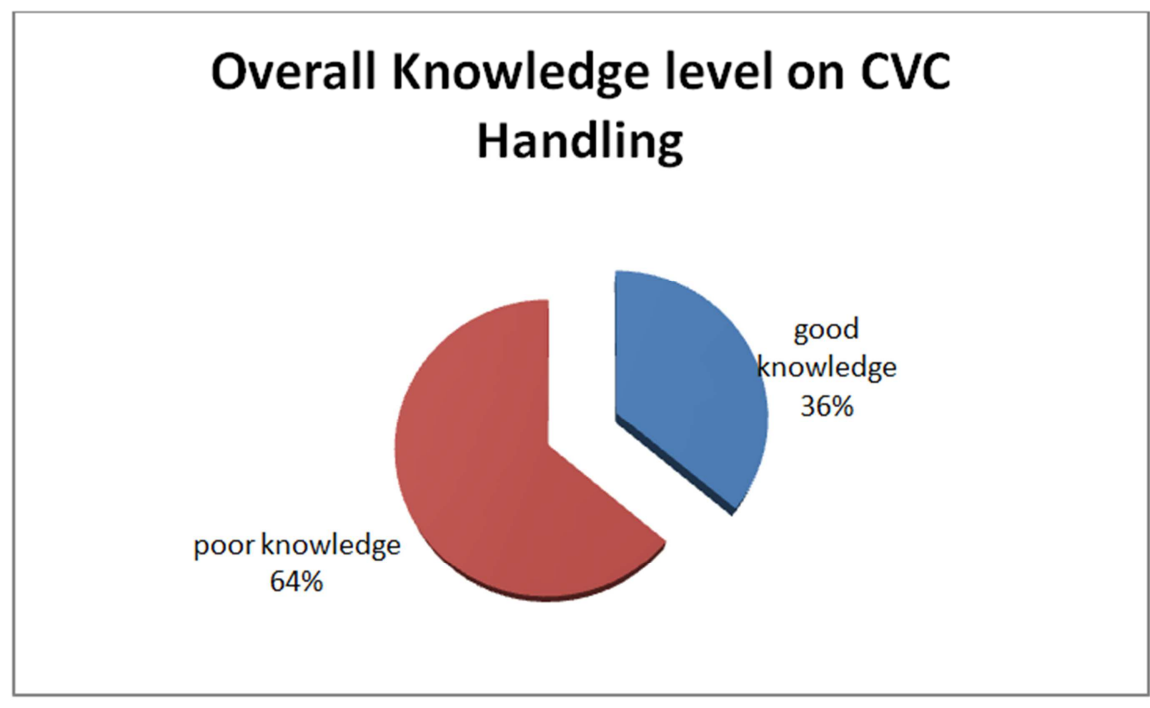

Figure 3. Overall knowledge level on CVC handling.

The overall knowledge level was computed against CCNs demographics and the results are displayed in table 5.

Table 5. Overall knowledge levels versus CCNs socio- demographic data.

\begin{tabular}{|c|c|c|}
\hline Variable/knowledge level & \%good knowledge & \%poor knowledge \\
\hline \multicolumn{3}{|l|}{ Age in years } \\
\hline $21-30$ & 18.8 & 81.2 \\
\hline $31-40$ & 41.7 & 58.3 \\
\hline $41-50$ & 44.4 & 55.6 \\
\hline $51-60$ & 0.0 & 100.0 \\
\hline \multicolumn{3}{|l|}{ Gender } \\
\hline male & 39.1 & 60.9 \\
\hline \multicolumn{3}{|l|}{ Highest education certificate } \\
\hline KRCHN & 25 & 75 \\
\hline KRCCN & 33.3 & 66.7 \\
\hline BSN & 47.4 & 52.6 \\
\hline \multicolumn{3}{|l|}{ Cadre } \\
\hline SNO & 38.8 & 61.2 \\
\hline NOIII & 25 & 75 \\
\hline \multicolumn{3}{|l|}{ Experience in $\mathrm{CCU}$} \\
\hline$<3$ years & 22.2 & 77.8 \\
\hline 3-6years & 43.6 & 56.4 \\
\hline 7-10years & 28.6 & 71.4 \\
\hline $10+$ years & 35.3 & 64.7 \\
\hline
\end{tabular}

Table 5 illustrates that the CCNs most knowledgeable on CVC handling were in age group 41-50 years. All CCNs aged 51-60 had poor knowledge on CVC handling as none of them scored $70 \%$ on overall knowledge. Male CCNs were more knowledgeable than female CCNs. BSNs were more knowledgeable on CVC handling than KRCHNs and KRCCNs. SNOs were more knowledgeable on CVC handling than other cadres. Those CCNs with 3-6 years' experience in $\mathrm{CCU}$ were more knowledgeable on CVC handling.

\subsubsection{Association of Nurse's Demographic Data and Knowledge Level on CVC Handling}

The test value used for computing P-values of $\mathrm{CCNs}$ demographic data was: age- 31-40 years, gender- female, highest certificate obtained- KRCCN, cadre- SNO, and experience in $\mathrm{CCU}-3-6$ years. The results are summarized in table 6 . 
Table 6. Association of nurse's demographic data and knowledge level on CVC handling.

\begin{tabular}{lllll}
\hline Demographic data & $\mathbf{X}^{2}$ & $d f$ & $\mathbf{r}^{2}$ & P-value \\
\hline Age (in years) & 5.539 & 3 & -.045 & .159 \\
Gender & .130 & 1 & .039 & .000 \\
Highest education & 1.469 & 2 & .129 & .001 \\
certificate obtained & & 3 & .086 & .000 \\
Cadre & 2.669 & 3 & .015 & .000 \\
Experience in CCU & 2.222 & 3 & \\
\hline
\end{tabular}

Table 6 shows that there was an association between gender and knowledge on $\mathrm{CVC}$ handling $\mathrm{P}<0.001$. The male gender had more knowledge on CVC handling. There was an association between highest education certificate and knowledge on $\mathrm{CVC}$ handling $\mathrm{P}=0.001$. BSNs who generally have higher education certificate than KRCHNs and KRCCNs were more knowledgeable on CVC handling.
There was an association between cadre and knowledge on CVC handling $\mathrm{P}<0.001$. SNOs who formed the highest cadre studied had more knowledge on CVC handling as compared to other cadres.

\subsection{Practice Level of CCNs in KNH CCUs on CVCs Handling}

Practice aspects on hand hygiene, skin antisepsis, prompt removal of unnecessary CVCs, proper CVC maintenance and changing IV administration system components were observed. Each critical care nurse was observed three times on three different patients while handling CVCs and the average score was obtained. Poor practice was interpreted as a score of below $70 \%$. The scores cut off was agreed upon by experts in critical care $(n=3)$. The results are summarized in table 7 .

Table 7. Practice level of CCNs in KNH CCUs on CVCs handling.

\begin{tabular}{|c|c|c|c|}
\hline \multirow{2}{*}{ Practice aspects } & \multirow{2}{*}{ Practice } & \multirow{2}{*}{$\begin{array}{l}\text { Frequency } \\
(\mathrm{n}=11)\end{array}$} & \multirow{2}{*}{$\begin{array}{l}\text { Percentage } \\
\text { (\%) }\end{array}$} \\
\hline & & & \\
\hline \multirow{2}{*}{$\begin{array}{l}\text { Uses alcohol-based hand rub solution or soap and water to perform hand hygiene prior to/ and after handling of } \\
\text { the CVC }\end{array}$} & Good & 8 & 72.7 \\
\hline & poor & 3 & 27.3 \\
\hline \multirow{2}{*}{$\begin{array}{l}\text { Cleans hands with alcohol-based hand rub solution or soap and water prior to any manipulation of the infusion } \\
\text { line }\end{array}$} & Good & 2 & 18.2 \\
\hline & poor & 9 & 81.8 \\
\hline Uses $2 \%$ chlorhexidine in $70 \%$ isopropyl alcohol to perform skin antisepsis during CVC dressing changes & poor & 11 & 100 \\
\hline \multirow{2}{*}{ Removes promptly unnecessary CVCs } & Good & 3 & 27.3 \\
\hline & poor & 8 & 72.7 \\
\hline \multirow{2}{*}{ Uses aseptic technique (sterile conditions) for all access to the CVC } & Good & 1 & 9.1 \\
\hline & poor & 10 & 90.9 \\
\hline Uses sterile transparent semipermeable dressing or sterile gauze to cover CVC insertion site & Good & 11 & 100 \\
\hline Uses gauze dressing on the CVC insertion site if the site is oozing or the patient is diaphoretic & Good & 11 & 100 \\
\hline Replaces the CVC dressing when it becomes damp, visibly soiled or loosened. & Good & 11 & 100 \\
\hline \multirow{2}{*}{ Routinely replaces gauze dressing every 2 days } & Good & 1 & 9.1 \\
\hline & Poor & 10 & 90.9 \\
\hline Routinely replaces semipermeable dressing every 7 days & Poor & 11 & 100 \\
\hline $\begin{array}{l}\text { Uses } 2 \% \text { chlorhexidine in } 70 \% \text { isopropyl alcohol antiseptic to clean the access hub of CVC prior to accessing - } \\
\text { rubs the CVC hub for at least } 15 \text { seconds }\end{array}$ & Poor & 11 & 100 \\
\hline \multirow{2}{*}{ TPN/IVI set opened and connected to the CVC aseptically } & Good & 3 & 27.3 \\
\hline & Poor & 8 & 72.7 \\
\hline \multirow{2}{*}{ Labels the tubing with due date and time of removal. } & Good & 3 & 27.3 \\
\hline & poor & 8 & 72.7 \\
\hline Changes IVFs administration set as per care bundle recommendation & poor & 11 & 100 \\
\hline
\end{tabular}

Most of the CCNs (72.7\%) perform hand hygiene with alcohol based hand rub solution or soap and water prior to and after handling CVC. However, only $18.2 \%$ clean hands before any manipulation of the infusion line. $2 \%$ chlorhexidine in $70 \%$ isopropyl alcohol is not available in $\mathrm{KNH}$ CCUs hence CCNs use povidone iodine for skin antisepsis during $\mathrm{CVC}$ dressing changes. Only 27.3\% of CCNs remove promptly unnecessary CVCs. Majority of CCNs do not perform aseptic technique for all access to the CVC as only $9.1 \%$ of them had good practices on this aspect. All the CCNs use the recommended dressing materials and they always replace them when they become damp, loosened or visibly soiled. Only $9.1 \%$ of CCNs routinely replace gauze dressing every 2 days; the others do this daily. Also, they routinely replace semipermeable dressing daily instead of every seven days. Antiseptic containing 70\% isopropyl alcohol is not available in KNH CCUs for cleaning the access hub prior to accessing thus the CCNs do not scrub the CVC access hub for at least 15 seconds. Most of the CCNs do not open and connect the TPN/IVI set to the CVC aseptically as only $27.3 \%$ have good practices on this aspect. Only $27.3 \%$ of CCNs label the tubing with due date and time of removal. All the CCNs do not change IVFs administration set as per the care bundle recommendation (continuous/ intermittent/needless/Parenteral Nutrition/ blood).

\section{Discussion}

Prevalence of central line associated blood stream infections (CLABSI) 
A review of medical records of patients admitted in $\mathrm{KNH}$ CCUs in 2015 revealed that the prevalence of CLABSIs was $3.53 \%$. A Previous prospective study showed CLABSI prevalence rate of $12 \%$ [9]. This decrease of $70.58 \%$ is a significant decline and could be attributed to use of advanced technology in CCUs and CLABSI clinical practice guidelines use including use of maximum sterile barrier precautions during CVC insertion, selection of subclavian vein as the site of choice for CVC insertion and increased use of effective antimicrobials. Although a decline in prevalence has been ascertained, this CLABSI burden remains high as critically ill patients with CVCs are expected to be CLABSI free. Gram negative organisms with Klebsiella pneumoniae as the leading cause $(60 \%)$ were the commonest cause of CLABSI and this compares to previous studies [9, 12].

Patient characteristics that influence occurrence of CLABSI

The patient characteristics that influence occurrence of CLABSI put into consideration in this study were age, gender, underlying disease, length of hospitalization with CVC in situ, site of CVC insertion, parenteral nutrition use and systemic antimicrobial therapy use. The age of patients studied did not have any influence on CLABSI occurrence $\left(\mathrm{r}^{2}\right.$ $=0.038$ ). This could be because the sample population did not have extremes of ages. Also, gender of the patient did not have any influence in CLABSI occurrence in this study $\mathrm{X}^{2}=0.060$ ). Males and females had equal chances of having CLABSI. This is in contrary to studies done in Geneva which identified male gender as a characteristic linked to increased CLABSI risk $[4,13]$. The underlying diseases or condition was shown to increase the risk of CLABSI $\left(X^{2}=15.249\right)$. Although earlier studies revealed that hematological, immunological, cardiovascular and gastrointestinal diseases are associated with increased risk of CLABSI infection [14], this study revealed that neurological disorders $(70 \%)$ put the critically ill patient at increased risk of this infection. This is because most patients admitted in CCUs of $\mathrm{KNH},(80 \%)$ have neurological disorders resulting mainly from injuries and disease. Also, these patients were mostly those who were severely ill especially with severe head injury resulting from increased motorcycle accidents and this increased their likelihood of having multiple CVCs with multiple infusion lines and also prolonged use of these devices with prolonged hospitalization. This study revealed that an increase in CVC dwell time increased CLABSI risk $\left(\mathrm{r}^{2}=0.774\right)$. This can be explained by the fact that the longer the CVC is in situ the more the exposure to microorganisms and the increased chances of infection. These findings compares to an earlier study which showed that the daily CLABSI risk was very low in patients during the first 7 days of catheterization but doubled thereafter [15]. Parenteral nutrition use in this study was associated with increased CLABSI occurrence $(\mathrm{P}=$ 0.013). This could be attributed to the fact that these solutions foster microbial growth especially the lipid preparations, their prolonged use and prolonged use of their infusion lines. These findings compares to earlier ones which demonstrated that the risk of CLABSI was higher among parenterally fed patients [14].

Nursing care related factors: nurses' knowledge and practices on CVC use that impact on CLABSI occurrence

$\mathrm{CCNs}$ in $\mathrm{KNH}$ were knowledgeable on hand hygiene at $67.4 \%$. A good number of them $(72.7 \%)$ perform hand hygiene before handling $\mathrm{CVC}$ although most of them do not perform hand hygiene before manipulation of infusion line as only $18.2 \%$ had good practices on this aspect. Studies done in Switzerland demonstrated that hand hygiene decreased HAIs, CLABSIs among them; from a baseline of $16.9 \%$ to $9.9 \%$ [12] thus failure of CCNs in $\mathrm{KNH}$ CCUs to perform hand hygiene as required could partly explain why CLABSIs are still a burden here. Although $\mathrm{CCNs}$ in $\mathrm{KNH}$ CCUs were knowledgeable on skin antisepsis at $90.7 \%$, chlorhexidine is not available hence they use povidone -iodine. Studies had found that chlorhexidine lowered the risk of CLABSIs by $50 \%$ in comparison to the use of povidone-iodine [19]. Also, Chlorhexidine was demonstrated to be superior in the prevention of CLABSI as compared to povidone- iodine[18, 20] Thus, continued use of povidone-iodine could be attributed to continued existence of CLABSI burden in $\mathrm{KNH}$ CCUs. CCNs were knowledgeable on prompt removal of unnecessary CVCs at $83.7 \%$ but had poor practices on this aspect as only $27.3 \%$ of them perform this practice as recommended. Thus CVCs were left in place for increased periods of time and this contributed to sustained CLABSIs burden in $\mathrm{KNH}$ CCUs. These findings compare to others which found that $4.8 \% \mathrm{CVC}$ days were unnecessary, and $4.6 \%$ catheter days were not justified respectively [23, 24]. $\mathrm{CCNs}$ are generally not knowledgeable on proper maintenance of CVCs as $14 \%$ of them were rated good knowledge and have poor practices in this aspect. Also, they are not knowledgeable on current guidelines in changing intravenous administration system components and thus do not practice these aspects as per recommendations. This leads to continued burden of CLABSI in KNH CCUs.

\section{Conclusion}

The researcher concludes that the prevalence of CLABSIs at $\mathrm{KNH}$ CCUs is $3.53 \%$. Neurological disorders as the underlying disease, increased length of hospitalization with CVC in situ, and parenteral nutrition use form the patient predictors/ characteristics/risk factors of critically ill patients with CLABSI in KNH CCUs. Nursing care related factors that predispose critically ill patients to CLABSIs in $\mathrm{KNH}$ CCUs are poor practices on hand hygiene before manipulation of infusion line, failure to remove unnecessary CVCs promptly, poor knowledge and practices on CVC maintenance and inadequate knowledge and outdated practices on changing intravenous administration system components.

The researcher recommends that at the CCUs level, CCNs should be informed on current proper CVC maintenance practices and IV administration system components changing via continued professional development and strong systems put in place to monitor, promote and support correct 
practices. Daily reviews of the continued requirement for CVCs should be carried out during patient care rounds with the multidisciplinary team and documented as part of patient's orders. Patients with neurological disorders and those on parenteral nutrition with a CVC in situ need to be monitored closely to prevent CLABSI occurrence. At the ward management level, hand hygiene should be incorporated into routine $\mathrm{CVC}$ handling procedures and strong systems put in place to support, monitor, and promote the correct behavior. At the institutional level, Chlorhexidine should be availed for skin antisepsis. CLABSI checklists should be availed and used on all patients on parenteral nutrition and those with Neurological disorders. At the policy level, policies and procedures on effective infection control measures on CLABSI should be developed and disseminated for use in CCUs and other settings.

The researcher also recommends further studies to be done on pediatric patients and non- CCU settings as this study left these out making it difficult to generalize the results to all patients with CVCs. Studies on other health care providers like doctors whose practices on CVC insertion and handling might contribute to development of CLABSI should be embarked on. Prospective studies using primary data sources to determine CLABSI prevalence should be carried out to provide an accurate picture of the prevalence currently on the ground. Also, studies on the impact of structures; institutional policies, procedures and practices on CLABSI occurrence should be undertaken.

\section{References}

[1] Centers for Disease Control and Prevention (2013). National Healthcare Safety Network. Device-Associated Module. Protocol and Instructions: Central Line- Associated Bloodstream Infection Event. http://www.cdc.gov/nhsn/psc_da.html.

[2] Higuera F, Rosenthal VD, Duarte P, Ruiz J, Franco G, Safdar $\mathrm{N}$, (2007). The effect of process control on the incidence of central venous catheter-associated bloodstream infections and mortality in intensive care units in Mexico. Journal of Critical Care Medicine. 33(9):2022-2027.

[3] Edgeworth J., (2009). Intravascular catheter infections. Journal of Hospital Infection. 73(4):323-330.

[4] Zingg W, Sax H, Inan C, Cartier V, Diby M, Clergue F, Pittet D, Walder B., (2008). Hospital-wide surveillance of catheterrelated bloodstream infection: From the expected to the unexpected. Journal of Hospital Infection. 73(1):41-46.

[5] Gurses AP, Seidl KL, Vaidya V, Bochicchio G, Harris AD, Hebden J, Xiao Y., (2008). Systems ambiguity and guideline compliance: A qualitative study of how intensive care units follow evidence-based guidelines to reduce healthcareassociated infections. Quality and Safety in Health Care Journal. 17(5):351-359.

[6] Rosenthal VD., (2009). Central line-associated bloodstream infections in limited-resource countries: A review of the literature. Journal of Clinical Infectious Diseases. 49(12):1899-1907.
[7] European Commission (2008). Communication from the Commission to the European Parliament and the Council on Patient Safety, Including Prevention and Control of Healthcare-Associated Infections. Commission of the European Communities.

[8] World Health Organization (2009). WHO Guidelines on Hand Hygiene in Health Care.

[9] Seko M, (2007). The Prevalence of Central Line Associated Infections at the Intensive Care Units of Kenyatta National Hospital. The University of Nairobi Research Archive. (Thesis document).

[10] The Joint Commission (2012). Preventing Central LineAssociated Bloodstream Infections: A Global Challenge, a Global Perspective. Oak Brook, Illinois.

[11] Rosenthal VD., Lynch P, Jarvis WR., (2011). Socioeconomic impact on device-associated infections in limited resource neonatal intensive care units: Journal of International Nosocomial Infection Control Consortium. 39(5):439-450.

[12] Allegranzi B, Bagheri Nejad S, Combescure C, Graafmans W, Attar H, Donaldson L, Pittet D., (2011). Burden of endemic health-care associated infection in developing countries: Systematic review and meta-analysis. The Lancet Journal 377(9761):228-241.

[13] Kritchevsky SB, Braun BI, Kusek L, Wong ES, Solomon SL, Parry MF, Richards CL, Simmons B, (2008). Evaluation of Processes and Indicators in Infection Control Study Group. The impact of hospital practice on central venous catheter associated bloodstream infection rates at the patient and unit level: A multicenter study. American Journal of Medicine. 23(1):24-38.

[14] Mollee P, Jones M, Stackelroth J, van Kuilenburg R, Joubert W, Faoagali J, Looke D, Harper J, Clements A., (2011). Catheter-associated bloodstream infection incidence and risk factors in adults with cancer: A prospective cohort study. Journal of Hospital Infection. 78(1):26-30.

[15] Niedner MF., (2010). National Association of Children's Hospitals and Related Institutions Pediatric Intensive Care Unit Patient Care FOCUS Group. The harder you look, the more you find: Catheter-associated bloodstream infection surveillance variability. American Journal of Infection Control. 38(8):585-595.

[16] Almuneef MA, Memish ZA, Balkhy HH, Hijazi O, Cunningham G, Francis C., (2005). Rate, risk factors and outcomes of catheter-related bloodstream infection in a paediatric intensive care unit in Saudi Arabia. Journal of Hospital Infections. 62(2):207-213.

[17] O'Grady NP, Alexander M, Burns LA, Dellinger EP, Garland J, Heard SO, Lipsett PA, Masur H, Mermel LA, Pearson ML, Raad II, Randolph AG, Rupp ME, Saint S; Healthcare Infection Control Practices Advisory Committee (HICPAC) (2011). Guidelines for the prevention of intravascular catheterrelated infections. Journal of Clinical Infectious Diseases. 52(9):e162-193.

[18] Mimoz O, Pieroni L, Lawrence C, Edouard A, Costa Y, Samii K, Brun-Buisson C. (2007). Prospective, randomized trial of two antiseptic solutions for prevention of central venous or arterial catheter colonization and infection in intensive care unit patients. Journal of Critical Care Medicine. 24(11):181823. 
[19] Chaiyakunapruk N, Veenstra DL, Lipsky BA, Saint S., (2002). Chlorhexidine compared with povidone-iodine solution for vascular catheter-site care: A meta-analysis. Annals of Internal Medicine Journal. 136(11):792-801.

[20] Milstone AM. Tamma PD, Aucott SW., (2010). Chlorhexidine use in the neonatal intensive care unit: Results from a national survey. Journal of Infection Control and Hospital Epidemiology. 31(8):846-849.

[21] Pratt RJ, Pellowe CM, Wilson JA, Loveday HP, Harper PJ, Jones SR, McDougall C, Wilcox MH., (2007). National evidence-based guidelines for preventing healthcareassociated infections in NHS hospitals in England. Journal of Hospital Infection. 65 Suppl 1:S1-64.

[22] Mermel LA., (2009). Prevention of intravascular catheter- related infections. Annals of Internal Medicine. 132(5), 391402.

[23] Zingg W, Walder B, Pittet D., (2011). Prevention of catheterrelated infection: Toward zero risk? Journal of Current Opinion in Infectious Diseases. 24(4):377-384.

[24] Trick WE, Vernon MO, Welbel SF, Wisniewski MF, Jernigan JA, Weinstein RA., (2008). Unnecessary use of central venous catheters: The need to look outside the intensive care unit. Journal of Infection Control and Hospital Epidemiology. 25(3):266-268

[25] Infusion Nurses Society (2016). Infusion Nursing Standards of Practice. Journal of Infusion Nursing. Jan-Feb;39Suppl 1:S164. 